\title{
Capacity Analysis of Selection Cooperation in Wireless Ad-Hoc Networks
}

\author{
Yanli Xu, Ping Wu, Lianghui Ding, and Lianfeng Shen
}

\begin{abstract}
In this letter, we analyze the transmission capacity of selection cooperation (SC) in wireless ad-hoc networks. We first derive the distribution of decoded relay set which follows inhomogeneous Poisson point process (PPP) and then present the closed-form expressions for the outage probability and transmission capacity. Furthermore, we propose an upper bound of source node density given an outage probability constraint and find an optimal transmitter density at which the transmission capacity reaches its maximum. Numerical results show that the performance gain provided by SC can be optimally adjusted by selecting appropriate node density. It is also shown that SC further expands advantage at severer large-scale fading.
\end{abstract}

Index Terms-Selection cooperation, interference, outage probability, transmission capacity, stochastic geometry analysis.

\section{INTRODUCTION}

$\mathbf{S}$ ELECTION cooperation (SC) is an attractive alternative of the cooperative schemes, wherein each source is paired with a best relay chosen from a set of potential ones [1]. It has been proved that $\mathrm{SC}$ can achieve full-order spatial diversity while avoiding the reduction of spectral efficiency compared to multiple-relay cooperation. The performance analysis of SC in a point to point system has been done rather extensively [2], [3].

However, these analyses are insufficient when SC is applied in large-scale wireless networks. On the one hand, interference is non-ignorable in an overlaid wireless network. On the other hand, the topology is random due to the mobility, death and new arriving of nodes which leads to random large-scale fading. Thus, to evaluate the advantage of SC, we need to analyze the average performance by considering both interference and random distances between transmitters. Stochastic geometry [4] provides a natural way of defining and computing macroscopic properties of large-scale networks by averaging over all potential geometrical patterns of nodes. Based on this theory, several works have been done to extend the investigation from a point to point system to a network. Outage performance and transmission capacity are presented for the case of non-cooperation communication in [5], [6]. For cooperation case, the transmission capacity is investigated in [7] without relay selection. In [8], a relay selection region

Manuscript received July 8, 2011. The associate editor coordinating the review of this letter and approving it for publication was I. R. Chen.

This work is supported in part by VINNOVA Sweden (grant 2008-00954), by the National Natural Science Foundation of China (60872004), and by the Research Fund of NCRL, Southeast University (2011A08).

Y. Xu and L. Shen are with the National Mobile Communications Research Laboratory, Southeast University, Nanjing, China 210096 (e-mail: \{yanlixu, lfshen\}@seu.edu.cn).

P. Wu is with Signals and Systems, Dept. of Engineering Sciences, Uppsala University, Uppsala, Sweden, 75121 (e-mail: ping.wu@angstrom.uu.se).

L. Ding is with the Dept. of Electronic Engineering, Shanghai Jiao Tong University, Shanghai, China 200240 (e-mail: 1hding @ieee.org).

Digital Object Identifier 10.1109/LCOMM.2011.090611.111475 is presented which is based on a uniformly distributed relay set for each source. This assumption can not guarantee that a best relay will be selected.

In this letter, we first derive the distribution of decoded relay set and then we present closed-form expressions for the average outage probability and transmission capacity of SC. Finally, upper bound source node density is presented given a tolerant outage probability. And an optimal density is also provided to achieve maximum performance gain via SC.

\section{SySTEM MOdel}

We consider an ad-hoc wireless network consisting of a large number of transmitters distributed over a large plane. We assume that the number of transmitters follows a homogenous PPP at a snapshot in time. This assumption is roughly equivalent to that transmitting nodes are independently and uniformly distributed, which is often reasonable for networks with indiscriminate node placement or substantial mobility. Transmitters select themselves as source nodes or potential relay nodes with some fixed probabilities. According to the thinning theory of Poisson process [9], the distributions of source nodes and potential relay nodes still follow homogenous PPPs, which are denoted by $\Theta\left(\rho_{s}\right)$ and $\Theta\left(\rho_{r}\right)$ with node densities $\rho_{s}$ and $\rho_{r}$ respectively. The set of receivers is disjoint with that of transmitters. Each source node has a unique intended receiver at a distance $d_{0}$. For easy explanation, we consider a source node $S$ located at the origin and its associated destination $D$. This pair serves as the reference communications link. The statistical property of this communication link can also be applied to others due to stationary [9].

Signals are subject to large-scale path loss proportional to $d^{-\alpha}$ for distance $d$ and exponent $\alpha>2$ as well as small scale fading following Rayleigh distribution. Hence the fading power factor $H_{i j}$ between nodes $i$ and $j$ follows exponential distribution with unit mean. Let $d_{i j}$ be the distance, then the received energy at $j$ from transmitter $i$ is

$$
\mathcal{E}_{i j}=\mathcal{E} H_{i j} d_{i j}^{-\alpha}
$$

where $\mathcal{E}$ is the transmission energy. Then the interference at receiver $j$ of source $S$ is

$$
I=\sum_{i \in \Theta\left(\rho_{s}\right), i \neq S} \mathcal{E}_{i j}=\mathcal{E} \sum_{i \in \Theta\left(\rho_{s}\right), i \neq S} H_{i j} d_{i j}^{-\alpha}=\mathcal{E} I_{\Theta\left(\rho_{s}\right)}
$$

where $I_{\Theta\left(\rho_{s}\right)}=\sum_{i \in \Theta\left(\rho_{s}\right), i \neq S} H_{i j} d_{i j}^{-\alpha}$ is the integrated noise. Here we consider an interference limited network and neglect the thermal noise, then the signal to interference ratio (SIR) at $j$ is

$$
S I R=\frac{\mathcal{E} H_{S j} d_{S j}^{-\alpha}}{I}=\frac{H_{S j} d_{S j}^{-\alpha}}{I_{\Theta\left(\rho_{s}\right)}}
$$




\section{PERFORMANCE ANALYSiS}

The transmission of the Decode-and-Forward SC strategy is divided into two time slots. Similar to [3], in the first slot, the source node transmits its data, then a best relay is selected by competition from the decoded set where nodes can decode data from the source correctly. Note that a node is possible to be selected as a relay by more than one source but this case happens with a small possibility and is thus ignored like in many previous works [8]. In the second time slot, the selected relay forwards data to the destination. Concerning the outage probability, we have the following proposition.

Proposition 1 Given the set of receivers $\Psi=D \cup \Theta\left(\rho_{r}\right)$, the outage probability can be expressed as

$$
P_{\text {out }, S j}=1-\exp \left(-c \rho_{s} d_{S j}^{2}\right) \quad \forall j \in \Psi
$$

where $c=\pi \kappa \eta_{t h}^{m}, \kappa=m \Gamma(m) \Gamma(1-m)$ and $m=2 / \alpha$. When $j=D$, we obtain the outage probability for direct transmission (DRT).

Proof: The outage probability is the probability that the SIR at the receiver falls below a specified threshold $\eta_{t h}$ required for successful reception. For equality, the transmission energy of source in DRT is $2 \mathcal{E}_{0}$. The transmission energy of source and relay in $\mathrm{SC}$ is $\mathcal{E}_{0}$ respectively. From (3), we can notice that SIR is independent of the transmission energy. Thus

$$
\begin{aligned}
& P_{\text {out }, S j}=\operatorname{Pr}\left\{\frac{H_{S j} d_{S j}^{-\alpha}}{I_{\Theta\left(\rho_{s}\right)}}<\eta_{t h}\right\} \\
& =1-E\left[\exp \left(-\eta_{t h} d_{S j}^{\alpha} I_{\Theta\left(\rho_{s}\right)}\right)\right] \\
& =1-F\left(\eta_{t h} d_{S j}^{\alpha}\right)
\end{aligned}
$$

where $E(\cdot)$ and $F(\cdot)$ are expect value and Laplace transform for random variable $I_{\Theta\left(\rho_{s}\right)}$, respectively. The Laplace transform of $I_{\Theta\left(\rho_{s}\right)}$ is [6]

$$
F(w)=\exp \left(-\pi \rho_{s} \kappa w^{m}\right)
$$

Substituting $F\left(\eta_{t h} d_{S j}^{\alpha}\right)=\exp \left(-\pi \rho_{s} \kappa \eta_{t h}^{m} d_{S j}^{2}\right)$ into (5) completes the proof.

After receiving data from $S$, potential relay nodes in $\Theta\left(\rho_{r}\right)$ who can decode data correctly constitute the decoded set $\mathcal{D}_{s}$. We provide the distribution of $\mathcal{D}_{s}$ in the following proposition.

Proposition 2 The number of nodes in set $\mathcal{D}_{s}$ follows an inhomogeneous PPP with the intensity measure $\Lambda(\mathcal{A})=$ $\int_{\mathcal{A}} \rho_{r} \exp \left(-\rho_{s} \kappa \eta_{t h}^{m} \sigma\right) d \sigma$, where $\mathcal{A} \subset \mathbb{R}^{2}$ is a closed area.

Proof: Define $\sigma$ as the area of the circle whose center is $S$ and radius is $r$ and let $d \sigma$ be infinitely small area on interval $[r, r+d r]$. According to (4), for a node $r$ away from $S$, i.e., $d_{S j}=r$, the probability it can decode data from $S$ correctly is $1-P_{\text {out }, S j}=\exp \left(-c \rho_{s} r^{2}\right)$. Thus the number of nodes in $d \sigma$ is $\rho_{r} \exp \left(-c \rho_{s} r^{2}\right) d \sigma$ which is depend on the location of node. Hence nodes in $\mathcal{D}_{s}$ follows an inhomogeneous PPP. For a closed area $\mathcal{A} \subset \mathbb{R}^{2}$, the average number of nodes decoding data correctly from $S$ is

$$
\Lambda(\mathcal{A})=\int_{\mathcal{A}} \rho_{r} \exp \left(-\rho_{s} \kappa \eta_{t h}^{m} \sigma\right) d \sigma .
$$

According to the SC strategy, the node in $\mathcal{D}_{s}$, that has instantaneous maximum signal strength at the receiver is selected as the relay for $S$, i.e, $R=\underset{j \in \mathcal{D}_{s}}{\arg \max }\left\{H_{j D} d_{j D}^{-\alpha}\right\}$. Then $R$ transmits data to $D$ interfered by the relays chosen for other sources. Let $\mathcal{D}_{i}$ denote the decoded set of source node $i$. Then the probability that a node $j \in \Theta\left(\rho_{r}\right)$ belongs to $\mathcal{D}_{i}$ is $P_{i j}=1-P_{\text {out }, i j}=\exp \left(-c \rho_{s} d_{i j}^{2}\right)$. Defining $\mathcal{D}=\bigcup_{i \in \Theta\left(\rho_{s}\right)} \mathcal{D}_{i}$ as the union set, we have $P^{\prime}=\sum_{i \in \Theta\left(\rho_{s}\right)} P_{i j}$, where $P^{\prime}$ is the total probability of $j \in \mathcal{D}$, i.e., the probability $j$ decoding data from at least one source correctly. Since $\Theta\left(\rho_{s}\right)$ is homogenous PPP, $P^{\prime}$ is independent of the location of $j$ based on the Slivnyak Theorem [9]. And $\mathcal{D}$ still follows homogenous PPP since it is obtained by thinning $\Theta\left(\rho_{r}\right)$ with probability $P^{\prime}$. Likewise, the number of chosen relays follows homogenous PPP. Since each source only selects one best relay, the density of chosen relays is also $\rho_{s}$. So we still denote the integrated interference induced by other chosen relays as $I_{\Theta\left(\rho_{s}\right)}$. With the help of the best relay $R$, the outage probability of SC is

$$
\begin{aligned}
P_{\text {out }, S C} & =\operatorname{Pr}\left\{H_{R D} d_{R D}^{-\alpha}<\eta_{t h} I_{\Theta\left(\rho_{s}\right)}\right\} \\
& =\operatorname{Pr}\left\{\max \left\{H_{j D} d_{j D}^{-\alpha}\right\}<\eta_{t h} I_{\Theta\left(\rho_{s}\right)}\right\}
\end{aligned}
$$

Defining the event $\digamma$ as

$$
\digamma=\left\{\max \left\{H_{j D} d_{j D}^{-\alpha}\right\}<\eta_{t h} I_{\Theta\left(\rho_{s}\right)}\right\}
$$

then the outage event can be expressed

$$
\begin{aligned}
\digamma & =\left\{\forall j \in \mathcal{D}_{s}, H_{j D} d_{j D}^{-\alpha}<\eta_{t h} I_{\Theta\left(\rho_{s}\right)}\right\} \\
& =\left\{\mathcal{D}_{s} \cap \widehat{A}=\emptyset\right\}
\end{aligned}
$$

where the set $\widehat{A}=\left\{\left(j \in \mathbb{R}^{2}\right) ; H_{j D} d_{j D}^{-\alpha} \geq \eta_{t h} I_{\Theta\left(\rho_{s}\right)}\right\}$.

As presented in proposition 2, the number of nodes in $\mathcal{D}_{s}$ follows inhomogeneous PPP. Thus, by making use of the avoidance probability we can express the outage probability for $\mathrm{SC}$ as follows

$$
P_{\text {out }, S C}=\exp \left\{-\int_{\mathbb{R}^{2}} 1_{\hat{\mathrm{A}}} \Lambda(d \sigma)\right\}
$$

where $1_{\hat{\mathrm{A}}}$ is the indicator function. Since

$$
\begin{aligned}
1_{\hat{\mathrm{A}}} & =\operatorname{Pr}\left\{H_{j D} d_{j D}^{-\alpha} \geq \eta_{t h} I_{\Theta\left(\rho_{s}\right)}\right\} \\
& =\exp \left(-\pi \rho_{s} \kappa \eta_{t h}^{m} d_{j D}^{2}\right) \\
& =\exp \left(-c \rho_{s} d_{j D}^{2}\right)
\end{aligned}
$$

then, (11) can be written as

$$
P_{\text {out }, S C}=\exp \left(-\int_{\mathbb{R}^{2}} \exp \left(-c \rho_{s} d_{j D}^{2}\right) \Lambda(d \sigma)\right)
$$

Substituting $\Lambda(d \sigma)=\rho_{r} \exp \left(-\rho_{s} \kappa \eta_{t h}^{m} s\right) d \sigma$ into (13) yields

$$
\begin{aligned}
& P_{\text {out }, S C}=\exp \left(-\rho_{r} \int_{0}^{2 \pi} d \theta \int_{0}^{\infty} r \exp \left(-c \rho_{s} d_{j D}^{2}\right) \exp \left(-c \rho_{s} r^{2}\right) d r\right) \\
& =\exp \left(-\rho_{r} \int_{0}^{2 \pi} d \theta \int_{0}^{\infty} r \exp \left(-c \rho_{s}\left(2 r^{2}+d_{0}^{2}-2 r d_{0} \cos \theta\right)\right) d r\right) \\
& =\exp \left(-\frac{\pi \rho_{r}}{c \rho_{s}} e^{-\frac{c \rho_{s} d_{0}^{2}}{2}}\right)
\end{aligned}
$$




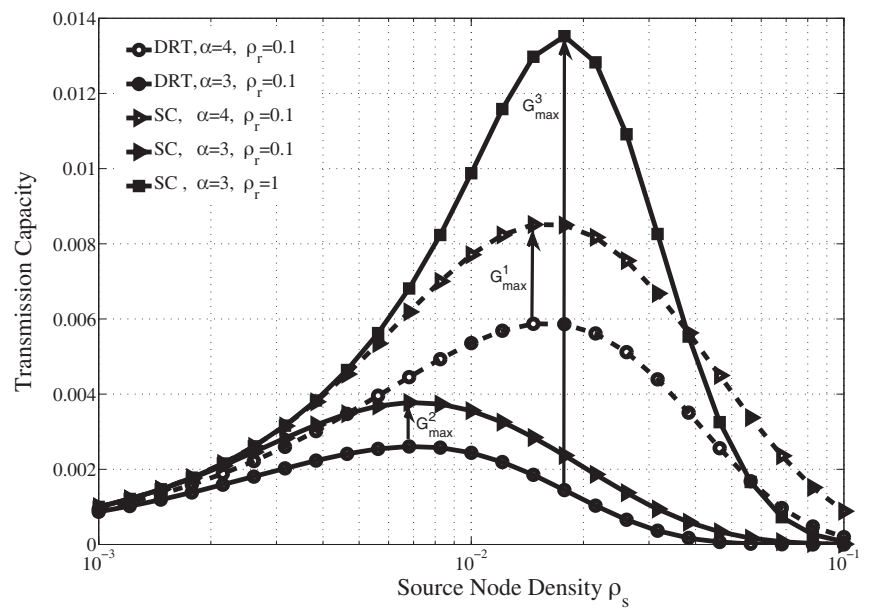

Fig. 1. Comparison of the transmission capacities of DRT and SC against source node density $\rho_{s}$.

From (14), we can find that $P_{\text {out }, S C}$ grows with $\rho_{s}$. Thus, given an outage constraint $\varepsilon$, i.e., $P_{\text {out }, S C} \leq \varepsilon$, there is an upper bound for $\rho_{s}$. In practice, the density of $\rho_{s}$ is small, so we use the approximation $e^{-\frac{c \rho_{s} d_{0}^{2}}{2}} \approx 1-\frac{c \rho_{s} d_{0}^{2}}{2}$. Hence, the upper bound of $\rho_{s}$ can be found from (14) as follows

$$
\overline{\rho_{s}}=\frac{\pi \rho_{r}}{c\left(\frac{\pi \rho_{r} d_{0}^{2}}{2}-\ln \varepsilon\right)}
$$

The transmission capacity is defined as the number of successful transmissions per unit area [10]. Given source node density $\rho_{s}$, we can obtain the transmission capacity of SC

$T C_{S C}=\rho_{s}\left(1-P_{\text {out }, S C}\right)=\rho_{s}\left(1-\exp \left(-\frac{\pi \rho_{r}}{c \rho_{s}} e^{-\frac{c \rho_{s} d_{0}^{2}}{2}}\right)\right)$

The outage probability of DRT can be obtained from proposition 1 . Then the transmission capacity of DRT is $T C_{D R T}=$ $\rho_{s} \exp \left(-c \rho_{s} d_{0}^{2}\right)$.

Proposition 3 Define the relay selection gain $G$ as the ratio of transmission capacity between SC and DRT, namely

$$
G\left(\rho_{s}\right)=\frac{T C_{S C}}{T C_{D R T}}=e^{c \rho_{s} d_{0}^{2}}\left(1-\exp \left(-\frac{\pi \rho_{r}}{c \rho_{s}} e^{-\frac{c \rho_{s} d_{0}^{2}}{2}}\right)\right)
$$

The maximum of relay selection gain $G$ is $G_{\max } \approx$ $e^{\frac{\pi \rho_{r} d_{0}^{2}}{\ln a}}\left(1-a^{-e^{-\frac{\pi \rho_{r} d_{0}^{2}}{2 \ln a}}}\right)$ and the corresponding optimal source node density to be $\rho_{s}^{o p t}=\frac{\pi \rho_{r}}{c \ln a}$, where $a=e^{\frac{\pi \rho_{r} d_{0}^{2}}{2}} / \pi \rho_{r} d_{0}^{2}$.

Proof: For a small $x$, we have $e^{-x} \approx 1-x$. Thus we approximate $G\left(\rho_{s}\right)$ as $e^{c \rho_{s} d_{0}^{2}}\left(1-e^{-\frac{\pi \rho_{r}}{c \rho_{s}}} e^{\frac{\pi \rho_{r} d_{0}^{2}}{2}}\right)$ for a small $\rho_{s}$. Letting $G^{\prime}\left(\rho_{s}\right)=0$, we can get the stationary point, i.e., the optimal source node density $\rho_{s}^{o p t}=\frac{\pi \rho_{r}}{c \ln a}$, where $a=e^{\frac{\pi \rho_{r} d_{0}^{2}}{2}} / \pi \rho_{r} d_{0}^{2}$. Substituting $\rho_{s}^{o p t}$ into (17), we obtain the maximum $G_{\max }$.

\section{Simulation Results}

Simulations are made on the transmission capacities of DRT and SC based on the closed-form expressions derived above.
Assuming that the distance between $S$ and $D$ is $d_{0}=2$ and the required threshold to decode data correctly is $\eta_{t h}=10 \mathrm{~dB}$, we study how the transmission capacity is affected by the parameters such as source node density $\rho_{s}$, potential relay node density $\rho_{r}$ and path loss factor $\alpha$.

Fig. 1 shows that the transmission capacities in all the cases first increase with $\rho_{s}$, and then decrease after reaching the maximum. That's because larger $\rho_{s}$ means more simultaneous transmitters while it also leads to higher level of interference. By comparing the curves of DRT (marked by circles) and $\mathrm{SC}$ (marked by triangles), we can see that SC brings gains over DRT and gains provided by SC are marginal at high $\rho_{s}$. Define $G_{\text {max }}^{1}$ and $G_{\text {max }}^{2}$ as maximum gains provided by SC when $\alpha$ is 4 and 3 respectively at $\rho_{r}=0.1$. Define $G_{\max }^{3}$ as the maximum gains when $\alpha=3, \rho_{r}=1$. We can see that these maximum gains can be achieved by choosing appropriate $\rho_{s}$. As for $\alpha$, we can notice that larger $\alpha$ (depicted by dotted lines) yields larger transmission capacity since the interfering signals are faded quickly with distance. SC further expands the advantage over DRT at higher $\alpha$. Comparing the curves of $\rho_{r}=0.1$ and $\rho_{r}=1$ for SC when $\alpha=3$, we can find that the transmission capacity increases with $\rho_{r}$ and this gain can be maximized by selecting $\rho_{s}$.

\section{CONCLUSiON}

We have analyzed the outage probability and transmission capacity of the decode-and-forward selection cooperation (SC) in ad-hoc wireless networks. It has revealed that SC enhances reliability and transmission capacity while this advantage is marginal at high source node density $\rho_{s}$. In addition, we can adjust $\rho_{s}$ and the potential relay node density $\rho_{r}$ to achieve target performance gain. In practice, it will be useful to adjust gains provided by SC and design cooperative networks.

\section{REFERENCES}

[1] E. Beres and R. Adve, "Selection cooperation in multi-source cooperative networks," IEEE Trans. Wireless Commun., vol. 7, pp. 118-127, Jan. 2008.

[2] D. Michalopoulos and G. Karagiannidis, "Performance analysis of single relay selection in Rayleigh fading," IEEE Trans. Wireless Commun, vol. 7, pp. 3718-3724, Oct. 2008.

[3] D. B. da Costa and S. Aissa, "Performance analysis of relay selection techniques with clustered fixed-gain relays," IEEE Signal Process. Lett., vol. 17, pp. 201-204, Feb. 2010.

[4] M. Haenggi, J. G. Andrews, F. Baccelli, O. Dousse, and M. Franceschetti, "Stochastic geometry and random graphs for the analysis and design of wireless networks," IEEE J. Sel. Areas Commun., vol. 27, pp. 1029-1046, Sep. 2009.

[5] S. Ye and R. S. Blum, "On the rate regions for wireless MIMO ad hoc networks," in Proc. IEEE Veh. Technol. Conf., vol. 3, pp. 1648-1652, Sep. 2004.

[6] A. M. Hunter, J. G. Andrews, and S. Weber, "Transmission capacity of ad hoc networks with spatial diversity," IEEE Trans. Wireless Commun., vol. 7, pp. 5058-5071, Dec. 2008.

[7] Z. Sheng, Z. Ding, and K. K. Leung, "Transmission capacity of decodeand-forward cooperation in overlaid wireless networks," in Proc. IEEE International Conference on Communications, May 2010, pp. 23-27.

[8] D. Li, C. Yin, C. Chen, and S. Cui, "A selection region based routing protocol for random mobile ad hoc networks," in Proc. IEEE Globecom, Dec. 2010, pp. 104-108.

[9] D. Stoyan, W. S. Kendall, and J. Mecke, Stochastic Geometry and its Applications, 2nd edition. Wiley, 1995.

[10] P. Gupta and P. R. Kumar, "The capacity of wireless networks," IEEE Trans. Inf. Theory, vol. 46, pp. 388-404, Mar. 2000. 